\title{
Time inhomogeneous Fokker-Planck equation for wave distributions in the abelian sandpile model
}

\author{
L. Anton* \\ Institute for Theoretical Physics, University of Stellenbosch, Private Bag X1, 7602 Matieland, South Africa \\ and \\ Institute of Atomic Physics, INFLPR, Lab 22, PO Box MG-36 R76900, Bucharest, Romania
}

(November 19, 2018)

\begin{abstract}
The time and size distribution of the waves of topplings in the Abelian sandpile model are expressed as the first arrival at the origin distribution for a scale invariant, time inhomogeneous Fokker-Planck equation. Assuming a linear conjecture for the the time inhomogeneity exponent as function of loop erased random walk (LERW) critical exponent, suggested by numerical results, this approach allows one to estimate the lower critical dimension of the model and the exact value of the critical exponent for LERW in three dimension. The avalanche size distribution in two dimensions is found to be the difference between two closed power laws.
\end{abstract}

05.10.Gg,04.40.-a

The abelian sandpile model (ASM) was introduced by Bak, Tang, and Wiesenfeld [1] as a minimal description for natural phenomena characterized by intermittent time evolution through events called avalanches which have scale invariant properties. The model is defined on a hypercubic lattice whose sites can accommodate a variable, positive number of grains. With a uniform distribution a site is chosen and its number of grains is increased by one. If its total number of grains exceeds a given critical threshold $z_{\max }$, the nearest neighbor sites increase their number of grains by one and the initial site loses the corresponding number of grains. Then the newly updated sites are checked for stability until there are no more unstable states. This event is an avalanche and the analytical properties of its distribution is still an open question [2, 3, [4, 5, 6]. Analytical approaches rely on the algebraic properties of the toppling rules and the decomposition of avalanches into simpler events called waves which are related to spanning trees on a lattice [2,7,9]. A wave of topplings is simply obtained by restraining the initial site, from where an avalanche was initiated, to topple again only after all the other unstable sites have relaxed. Recently it was shown that the wave distribution satisfies the finite size scaling ansatz, with critical exponents deduced from the equivalence between waves and spanning trees [2].

In this Letter we present numerical evidence that the critical exponents of the wave size and time distribution, which were deduced from geometrical considerations in [2, 7.9], can be related to the parameters of a scale invariant Fokker-Planck equation (FPE) in any dimension. In this way we make a connection between the geometrical properties of the waves and an evolution equation. As further confirmation of the validity of a FPE description for the abelian sandpile model, we show that the scaling behavior of the average number of unstable sites as function of time is predicted by the FPE. Using the expressions for the critical exponents deduced in [2] together with those inferred form the FPE approach we are able to find the lower critical dimension of the abelian sandpile model and, as an extra benefit, the exact value for the loop erased random walk (LERW) critical exponent $\nu$ in three dimensions. In two dimensions, the case in which the distribution of the last wave is known [9], we can compute the asymptotic behavior of the avalanche distribution with the result that it has the form of a difference between two close power laws. This has been previously proposed as an explanation for the failure of the finite size scaling approach [3,10].

The abelian sandpile model is, from its definition, a Markovian process whose states are specified by the lattice configuration. Once the initial point has been chosen randomly the dynamics of relaxation is deterministic, with the evolution determined by the initial configuration and the toppling rule.

We consider a coarse grain description of the sandpile evolution. Instead of a complete description, involving the number of grains at each site of the lattice, we use as variable the total number of unstable sites which exist at a given time, after an avalanche has started, irrespective of the configuration in which the sandpile is. This description is stochastic since the transition between two states with a given number of unstable sites depends on the configuration of the sandpile which is now taken randomly. Let us consider for this process the evolution equation describing the transition between states with different numbers of unstable sites: $P(t+1, n)=\sum_{n^{\prime}} W\left(t ; n, n^{\prime}\right) P\left(t, n^{\prime}\right)$; where $W\left(n, n^{\prime} ; t\right)$ is obtained by averaging over all the transitions between the configurations with the same number of unstable sites $n, n^{\prime}$ at a given time $t$. The transition probability $W\left(t ; n, n^{\prime}\right)$ may depend on time since the configuration of the unstable sites also depends on time; i.e. at the first step of a wave the unstable sites are some of the nearest neighbors of the initial site, eventually moving away. In this formulation the wave is equivalent to a particle performing a discrete random walk on the positive semi-axis with the transition probabilities depending 
on the particle position. An wave event is a first arrival at the origin problem, for the wave stops when all the sites, except the initial one, are stable. This random walk is close to a diffusion process since the number of unstable sites varies with bounded steps, $x(t+1)<2 D x(t)$, where $x(t)$ is the number of unstable sites and $D$ is the lattice dimension. From this analogy we expect that the distribution of first arrival at the origin, that is the wave duration, has a power law distribution as it has for the first return distribution of the simple diffusion process.

If we take the lattice size to infinity and the time unit to zero in an appropriate way the discrete Markov chain can be cast into a diffusion equation via the KramerMoyal expansion [12]. Here we shall not propose an explicit way to construct the FPE for the ASM, instead we use the fact that in the stationary state the sandpile is at criticality and we shall investigate the general FPE which yields critical behavior and has diffusion and drift coefficients behaving similarly to the sandpile model. Generically the FPE has the form $\partial_{t} p=-\partial_{x}[v(x, t) p]+(1 / 2) \partial_{x x}^{2}\left[D_{2}(x, t) p\right]$, where $p(x, t)$ is the probability density of the number of unstable sites, $x$ is the number of unstable sites, $t$ is the time since the wave has started. The drift coefficient $v(x, t)$ and the diffusion coefficient $D_{2}(x, t)$ are obtained by taking the continuum limit of the local first order moment $\sum_{j}\left(x_{i}-x_{j}\right) W(i, j ; t)$ and of the local second order moment $\sum_{j}\left(x_{i}-x_{j}\right)^{2} W(i, j ; t)$ [12]. Numerically we have found that the discrete diffusion coefficient $D_{2}(x, t)$ depends linearly on the number of unstable sites $x$. The slope depends on the dimension of the lattice but does not depend on the lattice size and the geometric condition (bulk or boundary wave)( see Fig. 11). Also we have found that the discrete drift coefficient $v(x)$ tends to a constant as the size of lattice grows, Fig. (1). The finite size effects affect the drift coefficient for the bulk waves at any value of the number of unstable sites, since the transition to states with larger number of unstable sites is smaller when the wave takes place near the boundary, while the statistics collects all the waves.

The simplest Fokker-Planck equation satisfying the scale invariance assumption and the numerical behavior of the discrete coefficients $D_{2}(x, t)$ and $v(x, t)$ is

$$
\partial_{t} p(x, t)=-\partial_{x}\left[v t^{-\alpha} p(x, t)\right]+\partial_{x x}^{2}\left[D_{2} x t^{-\alpha} p(x, t)\right]
$$

where $v, D_{2}, \alpha$ are constants. The initial condition for the above equation is $p\left(x, t=t_{0}\right)=\delta\left(x-x_{0}\right)$. Since we are interested in the time and size distribution for waves, which are first arrival events, we set an absorbing boundary condition at the origin $p(x=0, t)=0$, for the wave stops when the number of unstable sites, except for the initial one, is zero. The above differential equation is invariant under a scale transformation $x \rightarrow b x, \quad t \rightarrow b^{1 /(1-\alpha)} t$. We observe that we can eliminate the parameter $D_{2}$ by variable change $x \rightarrow D_{2} x$ and we change to $v \rightarrow v / D_{2}$.

Using a standard approach 14] one can find the asymp- totic behavior of the first arrival at the origin for Eq. (11): $P_{t}(t) \approx t^{-\tau_{t}}, \quad t \gg 1$ with

$$
\tau_{t}=1+(1-\alpha)|1-v|
$$

The second critical exponent we are interested in is the size distribution of waves. The size of the wave is the sum of the number of unstable sites until the wave stops; in the continuous formulation we have $s(t)=\int_{t_{0}}^{t} d t^{\prime} x\left(t^{\prime}\right)+$ $x\left(t_{0}\right)$.

We make the observation that the variable $s$ is a monotonic function of time as $\dot{s}=x(t)>0$. The relation between the two variables can be found using the average relation $\langle\dot{s}(t)\rangle=\langle x(t)\rangle$. Multiplying Eq. (1) by $x$ and integrating over $x$ we obtain that $\langle x(t)\rangle \approx t^{1-\alpha}, \quad t \gg 1$, where the average was normalized to the probability of surviving $\int d x p(x, t)$ until the moment $t$. Now we can use the variable $s$ in the time distribution of waves. We have $t^{-\tau_{t}} d t \approx s^{\frac{1}{2-\alpha}} d s^{\frac{1}{2-\alpha}}=s^{-\tau_{a}} d s$ for large $t$ and $s$, where

$$
\tau_{a}=1+\frac{(1-\alpha)}{2-\alpha}|1-v|
$$

This result can be checked easily by Monte Carlo simulation of a random walk constructed to be the discrete version of Eq. (1) or equivalent ones.

In Table f we show the values of the critical exponents $\tau_{a}, \tau_{t}$ taken from [2], using Eqs. (2,3) we have computed the values of the parameters $v$ and $\alpha$. We observe that $\alpha$ has the same value for bulk and boundary waves and the time inhomogeneity disappears at the critical dimension, $\alpha=0$ for $D=4$. Thus the exponent $\alpha$ can be interpreted as a measure of the correlation among the unstable sites bellow critical dimension.

Also, for $D=2$ we note that $\alpha$ has the same numerical value as the critical exponent characterizing the decay of the autocorrelation function of waves found in [4]. One more hint that the exponent $\alpha$ is related to the correlation on the lattice is that it depends linearly on the erased random critical exponent $\nu$ which has the values $\nu=4 / 5(D=2), \nu=0.616(D=3), \nu=1 / 2(D \geq 4)$ [2,11. Indeed one can see easily that the relation

$$
\alpha=\frac{4}{3}\left(\nu-\frac{1}{2}\right)
$$

holds exactly in $D=2$ and $D=4$ and has an error of 0.003 for $D=3$, case in which the critical exponent $\nu$ is only known from numerical simulation [11].

In the following we shall use Eq. (4), which holds for both bulk and boundary waves, as a conjecture for further exploration of the Table I. The fact that $\alpha$ has the same value for both bulk and boundary waves can be checked numerically using the first two moments $m_{n}(t)=\int x^{n} p(x, t)$ of the solution of Eq. (11). Integrating Eq. (1) over $x$ and using the absorbing boundary conditions at the origin, we obtain $m_{1}(t) / m_{0}(t) \approx t^{1-\alpha}$ which is independent of $v$. Numerical estimation of the above ratio in $D=2,3,4$, presented in Fig. (2), shows an 
excellent agreement with the predicted values (the error is less than 0.01).

In 22 it was shown that for waves the critical exponents for size and time distribution are given by

$$
\begin{aligned}
& \tau_{a}=2-\frac{1+\sigma}{d_{f}} \\
& \tau_{t}=1+\left(d_{f}-(1+\sigma)\right) \nu
\end{aligned}
$$

where $d_{f}$ is the fractal dimension of the wave, which has the values of the Euclidean dimension for $D=2,3,4$ and value 4 for higher dimension; $\sigma=1,0$ for bulk and boundary wave respectively.

Using the above proposed conjecture, Eq. (4), and Eqs. (2., 3, ए), from $(2-\alpha)\left(\tau_{a}-1\right)=\tau_{t}-1$ we obtain

$$
d_{f}=\frac{8}{3} \frac{1}{\nu}-\frac{4}{3} .
$$

This relation shows that that the minimum critical dimension of the abelian sandpile model is $4 / 3$ since the maximum value of $\nu$ is 1 . This result is in agreement with the observation that in $D=1$ the scaling in the abelian sandpile model breaks down 10 .

An additional benefit of the above relation is that it gives the value of the critical exponent for the loop erased random walk in three dimension. Indeed if we put $d_{f}=$ 3 , and keep in mind that $d_{f}=D$ for $D<4$, we get $\nu_{D=3}=8 / 13=0.615384 \ldots$ in perfect agreement with the numerical value found in [11]. In fact, assuming the conjecture Eq. (电) and identifying $D=d_{f}$, Eq. (6) yields a relation between the LERW critical exponent and space dimensionality.

Ktitarev et al [2] argue that all critical exponents of the abelian sandpile are determined by the critical exponent $\nu$. In order to complete this program we need the relation between the drift coefficient $v$ and $\nu$ and a relation between the the drift coefficients $v$ for the bulk and boundary waves. Using the Eq. (5) for $\tau_{t}$ together with Eqs. (2, 3) we obtain

$$
\frac{\left|1-v_{\text {bulk }}\right|}{\left|1-v_{\text {boundary }}\right|}=\frac{d_{f}-2}{d_{f}-1} .
$$

We need one more relation to connect the coefficients $\alpha$ and $v$ (bulk or boundary) with $\nu$. This can be obtained by using for example Eqs. (3. 4.5 ) from which we extract

$$
v_{\text {bulk }}=\left\{\begin{array}{lll}
\frac{6 \nu-3}{-4 \nu+5} & \text { if } \quad \nu \leq 4 / 5 \\
\frac{-14 \nu+13}{-4 \nu+5} & \text { if } \quad \nu>4 / 5
\end{array} .\right.
$$

We make the observation that the Eqs. (4, 4, 8) hold exactly in $D=3$ if we use the deduced value $\nu=8 / 13$, see Table I. At this point, we can conclude that we have found a self-consistent description of the critical properties for the time and size distribution of waves in ASM. The exponent $\alpha$ captures the lattice correlation and the drift $v$ controls the boundary condition of the wave, both of them being a function of the erased loop random walk critical exponent $\nu$ through the Eqs. (4. 7 8).
Now we are in the position to compute the asymptotic behavior for the avalanches in $D=2$ using the FPE. In this description an avalanche is the sum of a random number of waves. The waves are statistically independent being recurrent events [13]. This assumption might appear to be in contradiction with analysis of 4 but in fact in this approach the correlation is included already in the time inhomogeneity. When a wave of size $s$ touches the origin it has the the probability $p_{d}(s)$ to die, thus also concluding the avalanche, or a new wave can start with the probability $\left(1-p_{d}(s)\right)$. We choose the probability for the wave to die as $p_{d}(s)=s^{-3 / 8} \ln s$, so as to recover asymptotically the probability for the last wave: $p_{w}(s) p_{d}(s)=p_{l w}(s) \sim s^{-\frac{11}{8}}$ [8.96. The avalanche distribution can then be written as $p_{a}(s)=p_{w}(s) p_{d}(s)+$ $\int_{0}^{s} d s^{\prime} p_{w}\left(s^{\prime}\right)\left(1-p_{d}\left(s^{\prime}\right)\right) p_{w}\left(s-s^{\prime}\right) p_{d}\left(s-s^{\prime}\right)+\ldots$. We can sum the previous series after a Laplace transform in $s$ and we have

$$
p_{a}(\lambda)=\frac{p_{l w}(\lambda)}{1-\left(\left(1-p_{d}\right) p_{w}\right)_{\lambda}} .
$$

Applying again the Tauberian theorem [14] we find that asymptotically the avalanche size distribution behaves like

$$
p_{a}(s) \approx C_{1}(s \ln s)^{-1}+C_{2} s^{-\frac{11}{8}} .
$$

This kind of behavior has been proposed previously in the literature [10]. The fact that $1<11 / 8<2$ makes it difficult to obtain the 'pure' dominant behavior. From a numerical fit we obtain that $C_{2} / C_{1} \approx-0.25$, therefore $C_{1}(s \ln s)^{-1} \gg C_{2} s^{-\frac{11}{8}}$ for $s \geq 10^{6}$. Thus, the FPE approach predicts that the avalanche distribution in the bulk must have the same asymptotic behavior as the waves for very large values of $s$, provided that the statistics excludes the avalanches which are affected by the boundary.

In conclusion, we have used numerical hints to propose a FPE for the time and size distributions of the waves in the ASM. In this approach a wave is a first return event and the asymptotic properties of its distributions (time and size) are described by the first return probabilities of a time inhomogeneous FPE; the time inhomogeneity appears below the critical dimension $D=4$. Furthermore, this approach yields an analytical expression for the asymptotic behavior of the avalanche distribution in $D=2$ which goes beyond the finite size scaling hypothesis and in agreement with recent results [3,10].

Using the relation for the critical exponents $\tau_{a}, \tau_{t}$ deduced in [2] together with the the relations found through FPE approach and the conjecture (伍) we the propose explicit dependence of the critical exponents $\tau_{a}, \tau_{t}$ of the critical exponent of LERW $\nu$ (via $\alpha$ and $v$ ). A bonus of this approach is that it yields the value of the lower critical dimension, $4 / 3$, for the ASM and the exact value of $\nu=8 / 13$ in $D=3$ for LERW.

The author thanks H. B. Geyer, F. Scholtz, L. Boonaazier and A. van Biljon for useful discussions and H. B. Geyer for a critical reading of the manuscript. 
* email: anton@physics.sun.ac.za

[1] P. Bak, C. Tang, and K. Wiesenfeld, Phy. Rev. Lett. 59, 381 (1987).

[2] D. Ktitarev, S. Lübeck, P. Grassberger, and V. Priezzhev, Phys. Rev. E 61, 81 (2000), cond-mat/9907157.

[3] M. De Menech, A. L. Stella, and C. Tebaldi, Phy. Rev. E 58, 2677 (1998), cond-mat/9805045.

[4] M. De Menech, and A. L. Stella, unpublished,condmat/0002310.

[5] B. Drossel, cond-mat/9904075 v2 (unpublished).

[6] C. Tebaldi, M. De Menech, and A. L. Stella, Phy. Rev, Lett. 83, 3952,(1999), cond-mat/99032270.

[7] D. Dhar, Phy. Rev. Lett. 64, 1613 (1990).

[8] D. Dhar and S. .S. Manna, Phy. Rev. E 49, 2684 (1994).

[9] E. V. Ivashkevich, D. V. Ktitarev and, V. B. Priezzhev, Physica A 209, 347 (1994).

[10] D. Dhar, Physica A 263, 4 (1999).

[11] R. E. Bradley, S. Windwer, Phys. Rev. E 51, 241 (1995)

[12] H. Risken, The Fokker-Planck Equation (Springer-Verlang, Berlin Heidelberg, 1996).

[13] W. Feller, An Introduction to Probability Theory and Its Application (Wiley, New York, 1971), Vol. I.

[14] W. Feller, An Introduction to Probability Theory and Its Application (Wiley, New York, 1971), Vol. II; A. J. Bray, Phys. Rev. E 62, 103 (2000), cond-mat/9910135.

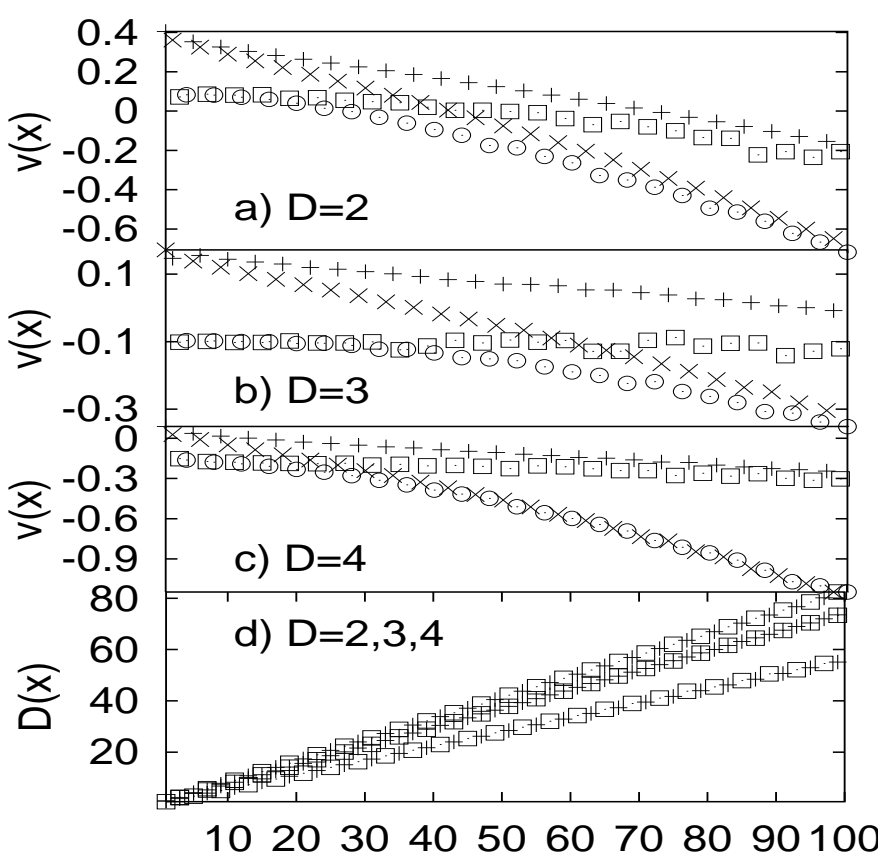

FIG. 1. The localnrifofolfinstatale sitesnd boundary wave in a) $D=2 ; L=1024(+), L=512(\times)$ for bulk waves and $L=1024(\square), L=512(\circ)$ for boundary waves; b) $D=3 ; L=128(+), L=64(\times)$ for bulk waves and $L=128(\square), L=64($ o) for boundary waves; c) $D=4$; $L=32(+), L=16(\times)$ for bulk waves and $L=32(\square)$, $L=16$ (o) for boundary waves; d)the local second order moment for bulk waves $(+)(D=2(L=1024), D=3(L=128)$, $D=4(L=32))$ and for boundary waves $\square(D=2(L=512)$, $D=3(L=64), D=4(L=16))$. 


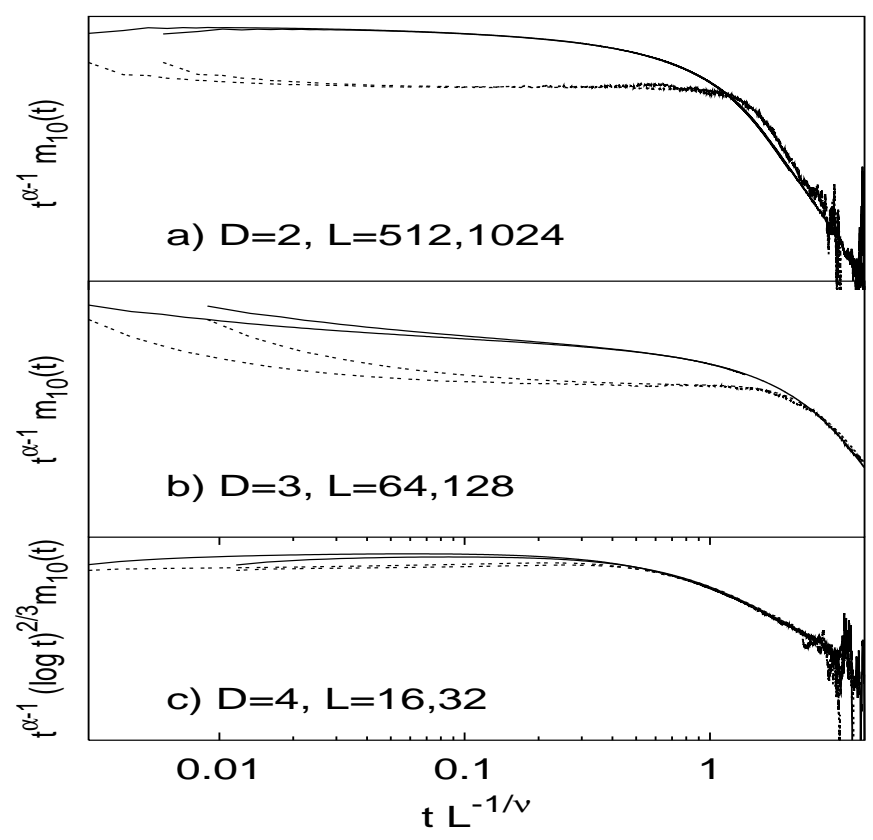

FIG. 2. Finite size scaling for the moment ratio $m_{10}(t)=m_{1}(t) / m_{0}(t)$ in: $D=2(\mathrm{a}), D=3(\mathrm{~b}), D=4$ (c) with continuous line for bulk waves and with dashed line for boundary waves. There is a good fit of the data with the exponents taken from Table (I). The logarithmic correction to scaling at $D=4$ has the same exponent as in Ref. [2].

TABLE I. The values for the critical exponent $\tau_{t}, \tau_{a}$ for the time and size distribution of waves taken from [2] together with the values of the exponent $\alpha$ and the drift coefficient $v$ computed from Eqs. (2.3). The last line shows the values of the erased loop random walk critical exponent $\nu$. For $D=3$ we show in parenthesis the values computed with the exact value $\nu=8 / 13$.

\begin{tabular}{llll}
\hline \hline $\mathrm{D}$ & 2 & 3 & 4 \\
\hline bulk & $\tau_{a}=1$ & $\tau_{a}=4 / 3$ & $\tau_{a}=3 / 2$ \\
& $\tau_{t}=1$ & $\tau_{t}=1.616(19 / 13)$ & $\tau_{t}=2$ \\
& $\alpha=2 / 5$ & $\alpha=0.152(2 / 13)$ & $\alpha=0$ \\
boundary & $v=1$ & $v=0.274(3 / 11)$ & $v=0$ \\
& $\tau_{a}=3 / 2$ & $\tau_{a}=5 / 3$ & $\tau_{a}=7 / 4$ \\
& $\tau_{t}=9 / 5$ & $\tau_{t}=2.232(29 / 13)$ & $\tau_{t}=5 / 2$ \\
& $\alpha=2 / 5$ & $\alpha=0.152(2 / 13)$ & $\alpha=0$ \\
$\nu$ & $v=-1 / 3$ & $v=-0.453(-5 / 11)$ & $v=-1 / 2$ \\
\hline \hline
\end{tabular}

